University of Nebraska - Lincoln

DigitalCommons@University of Nebraska - Lincoln

$10-15-2003$

\title{
Coarse-graining description of solid systems at nonzero temperature
}

Z.-B. Wu

University of Nebraska-Lincoln

Dennis J. Diestler

University of Nebraska-Lincoln, ddiestler1@unl.edu

Ruqiang Feng

University of Nebraska-Lincoln, rfeng1@unl.edu

Xiao Cheng Zeng

University of Nebraska-Lincoln, xzeng1@unl.edu

Follow this and additional works at: https://digitalcommons.unl.edu/chemzeng

Part of the Chemistry Commons

Wu, Z.-B.; Diestler, Dennis J.; Feng, Ruqiang; and Zeng, Xiao Cheng, "Coarse-graining description of solid systems at nonzero temperature" (2003). Xiao Cheng Zeng Publications. 31.

https://digitalcommons.unl.edu/chemzeng/31

This Article is brought to you for free and open access by the Published Research - Department of Chemistry at DigitalCommons@University of Nebraska - Lincoln. It has been accepted for inclusion in Xiao Cheng Zeng Publications by an authorized administrator of DigitalCommons@University of Nebraska - Lincoln. 


\title{
Coarse-graining description of solid systems at nonzero temperature
}

\author{
Z.-B. Wu \\ Department of Chemistry, University of Nebraska-Lincoln, Lincoln, Nebraska 68588 \\ D. J. Diestler \\ Department of Agronomy and Horticulture, University of Nebraska-Lincoln, Lincoln, Nebraska 68583 \\ R. Feng \\ Department of Engineering Mechanics, University of Nebraska-Lincoln, Lincoln, Nebraska 68588 \\ X. C. Zeng \\ Department of Chemistry, University of Nebraska-Lincoln, Lincoln, Nebraska 68588
}

(Received 19 May 2003; accepted 17 July 2003)

\begin{abstract}
The quasicontinuum (QC) technique, in which the atomic lattice of a solid is coarse-grained by overlaying it with a finite-element mesh, has been employed previously to treat the quasistatic evolution of defects in materials at zero temperature. It is extended here to nonzero temperature. A coarse-grained Hamiltonian is derived for the nodes of the mesh, which behave as quasiparticles whose interactions are mediated by the underlying (non-nodal) atoms constrained to move in unison with the nodes. Coarse-grained thermophysical properties are computed by means of the Monte Carlo (MC) method. This dynamically constrained QC MC procedure is applied to a simple model: A pure single crystal of two-dimensional Lennard-Jonesium. The coarse-grained isotropic stress $\left(\tau_{c}\right)$ is compared with the "exact" $\tau$ computed by the usual atomistic MC procedure for several thermodynamic states. The observed linear dependence of the error in $\tau_{c}$ on the degree of coarse-graining is rationalized by an analytical treatment of the model within the local harmonic approximation. (C) 2003 American Institute of Physics. [DOI: 10.1063/1.1607914]
\end{abstract}

\section{INTRODUCTION}

The behavior of solid systems with molecular defects (heterogeneities) involves coupled processes on spatial (and temporal) scales that span the gamut between molecular and macroscopic. In essence, an inhomogeneous region of molecular dimensions is coupled, through a "transition" zone, to a homogeneous region of macroscopic dimensions. Since the motions of the atoms in the inhomogeneous regions are coupled to those of atoms in the homogeneous regions, it would seem necessary to solve the many-body problem for the entire system in order to achieve an accurate description overall. Fully atomistic treatments by means of molecular dynamics (MD) and Monte Carlo (MC) have been applied to isolated defects in solids since the late 1960s. ${ }^{1-5}$ The fairly recent advent of parallel computing, to which molecular simulation is ideally suited, has permitted multi-million-atom MD studies of the behavior of defective crystals. In particular, investigations by researchers at the Louisiana State University ${ }^{6-8}$ and at $\mathrm{IBM}^{9,10}$ have revealed valuable insight into atomic mechanisms of microcracking in silicon.

Most systems of practical interest comprise orders of magnitude more than a million, or even a billion, atoms. It is practically impossible, even with the aid of the most powerful computers, to follow the detailed motions of all the atoms of such a large system. Since the atoms of the vast remote homogeneous regions are displaced only slightly from their (mean) equilibrium positions, continuиm mechanics should provide an adequate, if approximate, description of these regions. The persistent challenge is: How can we merge the accurate atomistic description necessary for the inhomogeneous regions with the continuum approximation that should suffice for the homogeneous regions, in order to achieve an overall description which is sufficiently accurate and computationally affordable?

Several efforts to combine atomistic and continuum descriptions of the behavior of materials have been made since the early 1970s. One approach ${ }^{11}$ is to partition the system into a "core" (inhomogeneous region) described atomistically and the "remainder" of the material (homogeneous region) treated as a continuum by means of the finite-element (FE) method. The core and remainder regions are coupled through a transition ("handshake") region, where the atoms and FE nodes are in one-to-one correspondence and move synchronously. In the transition region, the inner part uses the atomistic description and the outer part the FE method and each supplies boundary conditions for the other. This so-called FEAt method has been extended recently by Abraham et al. ${ }^{12,13}$ and by Broughton et al. ${ }^{14}$ to include an additional core whose atomistic dynamics is handled by the quantum-mechanical tight-binding (TB) approximation. The three levels of description are reconciled in TB-MD and MD-FE handshake regions. The technique has been applied to simulate the dynamics of crack propagation in silicon for durations up to $20 \mathrm{ps}$. To improve the coupling between MD and FE, Rudd and Broughton ${ }^{15}$ have proposed a coarsegrained MD description as an alternative to the FE method. The coarse-grained Hamiltonian differs from that of the FE method by a mesh-dependent correction term for thermal energy and reduces to the atomistic Hamiltonian when the 
mesh size decreases to atomic. However, by design coarsegraining precludes tracking explicit thermal motions of the atoms underlying the mesh. The accuracy of thermophysical properties computed by such a coarse-grained method, as indicated by the dependence of such properties on the size of finite elements, has not yet been thoroughly examined.

Another approach is based on the quasicontinuum (QC) method developed and refined by researchers at Brown University. ${ }^{16-20}$ Instead of partitioning the system into an atomistic core and its complementary continuum, they overlay the atomic lattice with an FE mesh and minimize the configurational (potential) energy as a function of the nodal coordinates under prescribed boundary conditions. In regions of small deformation remote from heterogeneities, large elements are used. The contribution of such a large element to the strain (configurational) energy is computed as if the (deformed) crystal lattice underlying the element were part of an infinite lattice homogeneously deformed in the same manner. The calculation thus requires only the (local) deformation of the large element. On the other hand, in the vicinity of heterogeneities the size of elements is greatly reduced. As a consequence the contribution of a small element to the strain energy involves not only the element itself, but also all neighboring elements that have underlying atoms within the range of interaction with atoms under the "central" element. The calculation is, therefore, highly nonlocal in that it requires knowledge of the deformations of the lattices underlying the many elements involved. In the limit where the size of the elements equals the lattice constant, the description becomes rigorously atomistic. In this way the multiple spatial scales are seamlessly coupled. The QC technique can be regarded as a coarse-grained description of the equilibrium state. It is particularly useful if one is primarily interested in the metastable behavior of a defective material (e.g., the degree of extension of a crack for a given increment of load) rather than the details of its temporal evolution (e.g., the propagation of a crack under critical load). However, the QC method is restricted to absolute zero $(T=0 \mathrm{~K})$.

We propose an extension of the QC technique to nonzero temperature. The key idea is to account for thermal effects by requiring nodal configurations in phase space to satisfy Boltzmann's law and computing the thermodynamic properties of the system statistically through ensemble averaging. However, as is the case with any coarse-graining procedure, QC coarse-graining, which converts the original atomistic Hamiltonian into a Hamiltonian for the FE nodes as quasiparticles, introduces constraints on the motions of atoms: All atoms underlying any given element move in concert with the nodes of that element. Therefore, the thermal motions of the original atoms are lost in coarse-graining. Nevertheless, thermal effects are partly accounted for by the nodal motions.

For the purpose of proof-of-principles, here we only carried out a statistical thermodynamic analysis of a highly idealized system: a single crystal of pure two-dimensional (2D) Lennard-Jonesium subjected to homogeneous isotropic deformation (see Sec. II). The coarse-grained Hamiltonian is derived in Sec. III. Section IV is devoted to the derivation of an explicit expression for the coarse-grained isotropic stress $\tau_{c}$. In Sec. V we detail the implementation of this expression in an $\mathrm{MC}$ computer program. We also present the results of MC simulations for several thermodynamic states. Comparison of coarse-grained results for $\tau_{c}$ with the "exact" results of standard atomistic MC simulations show that the error in $\tau_{c}$ is linear in the degree of coarse-graining, as well as the absolute temperature. In Sec. VI these linear dependencies are rationalized within the framework of the local harmonic approximation. $^{21-25}$

\section{DESCRIPTION OF MODEL SOLID}

We consider 2D Lennard-Jonesium, that is a pure monatomic substance for which the configurational (potential) energy $U$ can be expressed as a sum of two-body potentials

$$
\phi(r)=4 \epsilon\left[(\sigma / r)^{12}-(\sigma / r)^{6}\right],
$$

where $r$ is the distance between a pair of atoms. For a system comprising $N$ atoms we, therefore, have

$$
U\left(\mathbf{r}_{1}, \mathbf{r}_{2}, \ldots, \mathbf{r}_{N}\right)=\frac{1}{2} \sum_{i=1}^{N} \sum_{j \neq i}^{N} \phi\left(r_{i j}\right)
$$

where

$$
r_{i j}=\left|\mathbf{r}_{i}-\mathbf{r}_{j}\right|=\left[\left(x_{i}-x_{j}\right)^{2}+\left(y_{i}-y_{j}\right)^{2}\right]^{1 / 2},
$$

is the distance between atoms $i$ and $j$. The Hamiltonian is

$$
H=\sum_{i=1}^{N} \mathbf{p}_{i}^{2} / 2 m+U\left(\mathbf{r}_{1}, \mathbf{r}_{2}, \ldots, \mathbf{r}_{N}\right),
$$

where $\mathbf{p}_{i}$ is the momentum of atom $i$ conjugate to its position $\mathbf{r}_{i}$ and $m$ is the mass of an atom.

The hexagonal close-packed structure of the crystal is displayed in Fig. 1. The atoms are depicted as being in their equilibrium positions at $T=0 \mathrm{~K}$ for the given density. This is the reference configuration that we employ for the MC simulations described in Sec. V. To render the system finite, we impose periodic boundary conditions (p.b.c.) on pairs of lines parallel with $x$ and $y$ axes, as indicated in Fig. 1. These lines are so drawn that the rectangle formed encompasses exactly $2^{2 p+1}$ unit cells, each of which contains one atom. (For the illustration in Fig. 1, $p=3$.) There are $2^{p}$ unit cells along the $x$ direction and $2^{p+1}$ along the $y$ direction. Under an isotropic deformation, the dimensions of the system can be determined from the lattice constant $a$ (the nearestneighbor distance) and the number of atoms as

$$
\begin{aligned}
& L_{x}=a \sqrt{N / 2}, \\
& L_{y}=\sqrt{3} L_{x}, \\
& A=L_{x} L_{y}=\frac{\sqrt{3} a^{2}}{2} N .
\end{aligned}
$$

The number density, which we take as a measure of the (isotropic) deformation, is given by

$$
\rho=N / A=\frac{2}{\sqrt{3} a^{2}} .
$$

Gibbs' fundamental relation governing reversible transformations of the system can be written 


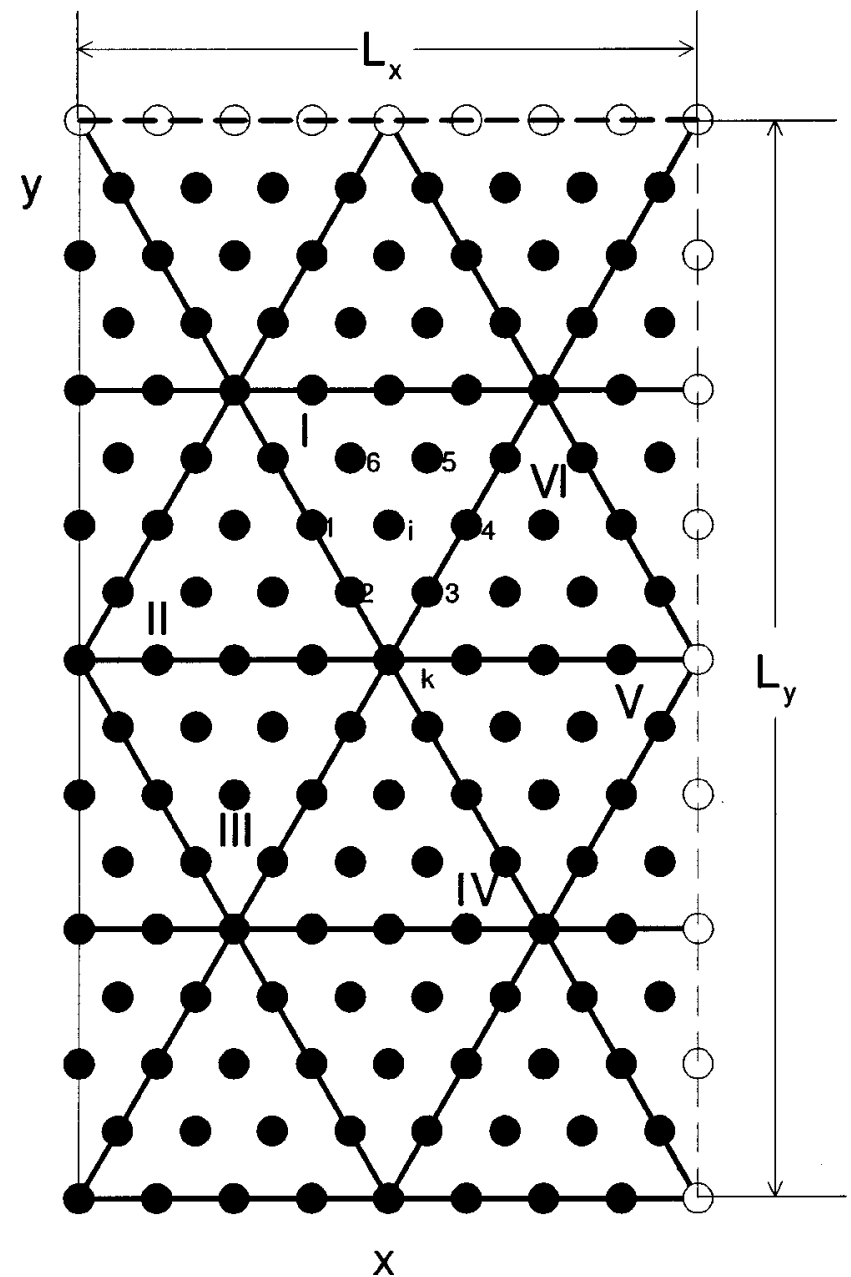

FIG. 1. Schematic of two-dimensional Lennard-Jonesium crystal in reference configuration. Filled circles represent atoms in their equilibrium positions at $T=0 \mathrm{~K}$. Periodic boundary conditions imposed on edges. Open circles stand for periodic images of atoms on opposite edge. $k$ denotes a particular node common to elements labeled with Roman numerals I-VI. Atom $i$ represents a "centroid" atom.

$$
d F=-S d T+\mu d N+d W_{\text {mech }},
$$

where $F$ stands for the Helmholtz energy, $S$ for the entropy and $\mu$ for the chemical potential. Taking into account only compression along axial directions, we can express the differential of the mechanical work done by the system on the surroundings as

$$
d W_{\text {mech }}=T_{x x} L_{y} d L_{x}+T_{y y} L_{x} d L_{y},
$$

where $T_{\alpha \alpha}(\alpha=x, y)$ is the mean force per unit length (stress) acting on the lines perpendicular to the $\alpha$ axis. By convention, if the force acts in the outward direction, $T_{\alpha \alpha}<0$, so that $d W_{\text {mech }}<0$ if the system expands $\left(d L_{\alpha}>0\right)$. Note that because the crystal lattice is centrosymmetric, $T_{x x}=T_{y y}$ for isotropic deformations of the system.

\section{COARSE-GRAINING TRANSFORMATION}

Suppose the crystal lattice in the reference configuration is covered with a mesh of triangular elements, as shown in Fig. 1. We assume, for convenience, that the nodes of the mesh coincide with atoms of the underlying lattice. We des- ignate the reference configuration by $\mathfrak{R}^{0}$, which stands for the collection of nodal positions $\left\{\mathbf{R}_{k}^{0}=\left(X_{k}^{0}, Y_{k}^{0}\right), k\right.$ $\left.=1,2, \ldots N_{n}\right\}$, where $N_{n}$ is the number of nodes of the mesh. We further assume that when an element $e$ is distorted (by displacing its nodes from their positions in $\mathfrak{R}^{0}$ ), the lattice underlying $e$ is deformed homogeneously. That is to say, after displacement of the nodes (so that the system is in a new configuration $\mathfrak{R}$ ), the configuration of atoms underlying $e$ can be regarded as an affine transformation ${ }^{26}$ of their configuration in $\mathfrak{R}^{0}$. The position $\mathbf{r}_{i}=\left(x_{i}, y_{i}\right)$ of atom $i$ can be written in terms of its position in $\mathfrak{R}^{0}$ as

$$
\mathbf{r}_{i}=\mathbf{m r}_{i}^{0}+\mathbf{c},
$$

where $\mathbf{r}_{i}$ and $\mathbf{r}_{i}^{0}$ are $2 \times 1$ matrices whose elements are the Cartesian components of the position of atom $i$ in $\mathfrak{R}$ and $\mathfrak{R}^{0}$, respectively, $\mathbf{m}$ is the $2 \times 2$ linear transformation matrix for deformation, and $\mathbf{c}$ is the $2 \times 1$ rigid-body motion matrix. Using the interpolation functions for the three-node triangular element, ${ }^{27}$ we can recast (9) in component form as

$$
r_{i \alpha}=\sum_{k=1}^{3} A_{i}(k) R_{k \alpha} / A_{e}, \quad \alpha=x, y,
$$

where $k$, a "local" label pertaining to element $e$, designates the nodes with positions $\mathbf{R}_{k}=\left(R_{k x}=X_{k}, R_{k y}=Y_{k}\right), A_{e}$ denotes the area of element $e$ (in the configuration $\mathfrak{R}$ ) and $A_{i}(k)$ denotes the area of the triangle inscribed in $e$ (in $\mathfrak{R}$ ), one of whose sides coincides with the side of element $e$ opposite node $k$ and one of whose vertices coincides with the point $\left(x_{i}, y_{i}\right)$.

From (10) we conclude that the positions of all atoms underlying a given element $e$ can be expressed in terms of the positions of only the three nodes of $e$. The number of independent variables needed to determine the configuration of the underlying atoms is thus vastly reduced, specifically from $2 N_{a}^{e}$ (where $N_{a}^{e}$ is the number of atoms that lie under $e$ ) to 6 . Therefore, we can represent the atomic configuration of the whole crystal succinctly by

$$
\mathbf{r}_{\alpha}^{(N)}=\mathbf{d R}_{\alpha}^{\left(N_{n}\right)}, \quad \alpha=x, y,
$$

where $\mathbf{r}_{\alpha}^{(N)}$ is the $N \times 1$ matrix of atomic $\alpha$ coordinates and $\mathbf{R}_{\alpha}^{\left(N_{n}\right)}$ is the $N_{n} \times 1$ matrix of nodal $\alpha$ coordinates. The elements of the $N \times N_{n}$ matrix $\mathbf{d}$ can be obtained from the relation (10). Since the coordinates of any given atom are determined completely by the coordinates of the three nodes of the element which it underlies, $\mathbf{d}$ is quite sparse. Defining the $2 N \times 2 N_{n}$ partitioned matrix

$$
\mathbf{D}=\left(\begin{array}{ll}
\mathbf{d} & \mathbf{0} \\
\mathbf{0} & \mathbf{d}
\end{array}\right),
$$

we can rewrite the two equations implicit in (11) as one

$$
\mathbf{r}=\mathbf{D R} \text {. }
$$

Here the first $N\left(N_{n}\right)$ elements of $\mathbf{r}(\mathbf{R})$ are $x$ coordinates of the atoms (nodes) and the second $N\left(N_{n}\right)$ elements of $\mathbf{r}(\mathbf{R})$ are $y$ coordinates of atoms (nodes). For ease of notation, we have dropped the superscripts indicating the dimensions of the column vectors. 
Corresponding to (13) the momenta conjugate to coordinates $\mathbf{r}$ can be expressed by

$$
\mathbf{p}=m \dot{\mathbf{r}}=m \mathbf{D} \dot{\mathbf{R}},
$$

where a dot over a symbol signifies the derivative with respect to time. Transforming to nodal coordinates $\mathbf{R}$ and conjugate momenta $\mathbf{P}$, we can cast the coarse-grained Hamiltonian compactly as

$$
H_{c}=\frac{1}{2 m} \mathbf{P}^{T}\left(\mathbf{D}^{T} \mathbf{D}\right)^{-1} \mathbf{P}+U(\mathbf{R}),
$$

where the superscript $T$ denotes the transpose. It is apparent from (15) that under the constraints on the coordinates and momenta [see (13) and (14)] introduced by coarse-graining, the original system of $N$ atoms is reduced to a system of $N_{n}$ nodes, which behave as quasi-particles.

\section{COARSE-GRAINED THERMOMECHANICAL PROPERTIES}

Since the controlled independent thermodynamic state variables are $T, N, L_{x}$, and $L_{y}$ [see (7) and (8)], we work in the canonical ensemble, whose characteristic function is the Helmholtz energy $F\left(T, N, L_{x}, L_{y}\right)$. The coarse-grained Helmholtz energy is expressible as

$$
F_{c}=-k_{B} T \ln Q_{c},
$$

where $Q_{c}$ is the coarse-grained (classical) canonical partition function

$$
Q_{c}=h^{-2 N_{n}} \int d \mathbf{P}^{N_{n}} \int d \mathbf{R}^{N_{n}} \exp \left(-H_{c} / k_{B} T\right) .
$$

In (16) and (17) $k_{B}$ and $h$ stand, respectively, for Boltzmann's constant and Planck's constant. To simplify the notation, henceforth we drop the superscript $N_{n}$. Then (17) can be rewritten explicitly

$$
\begin{aligned}
Q_{c}= & h^{-2 N_{n}} \prod_{i=1}^{N_{n}} \int_{-\infty}^{+\infty} d P_{i x} \int_{-\infty}^{+\infty} d P_{i y} \\
& \times \exp \left(-\mathbf{P}^{T} \mathbf{M}^{-1} \mathbf{P} / 2 k_{B} T\right) Z_{c} .
\end{aligned}
$$

Here, the mass matrix is defined by

$$
\mathbf{M}=m \mathbf{D}^{T} \mathbf{D},
$$

and the coarse-grained configuration integral $Z_{c}$ is defined by

$$
Z_{c}=\prod_{i=1}^{N_{n}} \int d \mathbf{R}_{i} \exp \left[-U\left(\mathbf{R}_{1}, \mathbf{R}_{2}, \ldots, \mathbf{R}_{N_{n}}\right) / k_{B} T\right],
$$

where the integrals over nodal positions extend over the domain of the crystal.

We focus especially on the diagonal elements of the stress tensor, which, according to (7) and (8) can be expressed as

$$
L_{\beta} T_{\alpha \alpha}=\left(\frac{\partial F}{\partial L_{\alpha}}\right)_{T, N, L_{\beta}}, \alpha, \beta=x, y ; \alpha \neq \beta .
$$

Using (16) and (18), we deduce from (21)

$$
L_{\beta} T_{\alpha \alpha}=-k_{B} T \frac{\partial \ln Z_{c}}{\partial L_{\alpha}} .
$$

Following the regular derivation, ${ }^{28}$ we obtain

$$
T_{\alpha \alpha}=-\frac{N_{n} k_{B} T}{A}+\frac{1}{A}\left\langle\sum_{k=1}^{N_{n}} \frac{\partial U}{\partial R_{k \alpha}} R_{k \alpha}\right\rangle, \quad \alpha=x, y,
$$

where the angular brackets denote the ensemble average.

Observe that the expression in (23) exhibits the usual virial form for the diagonal components of the stress tensor, ${ }^{28}$ except that it refers to $N_{n}$ quasiparticles (the nodes) instead of the original $N$ atoms. In the limit $N=N_{n}$, where every atom is a node of the mesh, then the expression for $T_{\alpha \alpha}$ is identical with the usual atomic expression. The first term in (23) is the ideal-gas contribution; the second term accounts for the effective interactions among the nodes, which are mediated by the underlying atoms that move in concert with those nodes. Both terms depend on $T$ as well as on the nodal density $N_{n} / A$.

The coarse-grained configurational (potential) energy can be written as

$$
U(\mathbf{R})=\sum_{e=1}^{N_{e}} U_{e}
$$

where the sum on $e$ is over the $N_{e}$ elements that cover the crystal (see Fig. 1). The contribution of element $e$, which depends implicitly on the configuration of nodes $\mathbf{R}$ (in $\mathfrak{R}$ ), is expressible as

$$
U_{e}=\int_{A_{e}} d^{2} r \rho_{e}(\mathbf{r}) \tilde{u}_{e}(\mathbf{r})
$$

in terms of the local density $\rho_{e}(\mathbf{r})$ and the local potential energy per atom $\tilde{u}_{e}(\mathbf{r})$ at $\mathbf{r}$. The symbol $\mathbf{R}$ now stands for the set of nodal coordinates. The exact calculation of $U_{e}$ would require a laborious evaluation of a double sum over pairwise atomic interactions [see (2)], but of course such a procedure would defeat the purpose of coarse-graining. Instead, following the treatment of Tadmor et al., ${ }^{16}$ we approximate $\widetilde{u}_{e}(\mathbf{r})$ by:

$$
\tilde{u}_{e} \simeq \frac{1}{2} \sum_{j \neq i} \phi\left(r_{i j}\right), \quad r_{i j}<r_{c}
$$

where $i$ denotes the "centroid" atom (i.e., the atom underlying $e$ which is nearest the centroid of $e$ ) and the sum is over all atoms $j$ which lie within a cut-off circle of radius $r_{c}$ centered on $i$, regardless of whether $j$ underlies $e$ or a neighboring element intersected by the cutoff circle. Under the assumption of uniform deformation, $\rho_{e}(\mathbf{r})=N_{a}^{e} / A_{e}$. Thus, the integrand in (25) does not depend on $\mathbf{r}$ and we have

$$
U_{e} \simeq N_{a}^{e} \tilde{u}_{e} .
$$

The errors implicit in (27) are ascribable to essentially two sources: (i) The use of a cutoff; (ii) the size of the element. By choosing $r_{c}$ sufficiently large, we can maintain the "cut-off" error at an acceptable level. Now assuming that the error due to source (i) is under control, we consider errors due to source (ii). We note first that if the deformation of the whole system were uniform, there would be no error depend- 
ing on the size of the element. Thus, if the element is sufficiently large to contain the cutoff circle (i.e., if the element is "local" in the parlance of Tadmor et al. $\left.{ }^{16}\right),(27)$ is accurate, as long as the deformation in the neighborhood of the element is uniform so that $\tilde{u}_{e}(\mathbf{r})$ is exactly equal to $\tilde{u}_{e}$ [see (26)]. Otherwise, $\tilde{u}_{e}(\mathbf{r})$ varies near the sides of the element, where atoms underlying the element interact with those underlying contiguous elements subject to different (uniform) deformations. The error due to neglect of this variation in $\tilde{u}_{e}(\mathbf{r})$ decreases with increasing size of the element, since the larger the element the more atoms underlying it which are out of range of interaction with atoms underlying neighboring elements. Even so, the sign of the error alternates between neighboring elements and hence this type (ii) error is compensated in $U(\mathbf{R})$, which is equal to the sum of $N_{a}^{e} \widetilde{u}_{e}$ over all elements. This suggests that one should avoid a drastic variation in element size over a small homogeneous region in order to minimize the error in $U(\mathbf{R})$.

For a small enough element the cutoff circle intersects several neighboring elements, thus rendering the element "nonlocal." 16 In this situation every atom underlying the small element interacts with atoms in the exterior (intersected) elements. Expression (27) remains accurate if the deformation within the cut-off circle is uniform, for the reason stated above. However, if the deformation is nonuniform (as expected where small elements are generally employed in the vicinity of a heterogeneity), then the accuracy of (27) increases with decreasing size of the element. This is because the "centroid" atom in a constant-strain element is least sensitive to variations in deformation of the neighboring elements. Hence, the larger the nonlocal element, the greater is the underestimate of the effect on $\widetilde{u}_{e}$ due to variation of the deformation in the vicinity of the element.

The approximation (27) is most accurate in the coarsegraining limit $N_{n}=N$, where every atom is a node and the elements are of atomic size. Note that in this limit there are $2 N$ elements, with $1 / 2$ atom underlying each. Thus, combining (24), (26), and (27), we have

$$
U(\mathbf{R})=\frac{1}{2} \sum_{e=1}^{2 N} \frac{1}{2} \sum_{j \neq i} \phi\left(r_{i j}\right) .
$$

The sum on $e$ in (28) can be replaced by a sum on centroid atoms $i$ to give

$$
U(\mathbf{R})=\frac{1}{2} \sum_{i=1}^{2 N} \frac{1}{2} \sum_{j \neq i} \phi\left(r_{i j}\right) .
$$

In the coarse-graining limit, the centroid atom is the nodal atom closest to the centroid. Provided that the index $i$ counts each atom just twice, then the double sum in (29) is precisely equivalent to that in (2), which is the exact expression for the configurational energy of the crystal. The coarse-graining limit is essentially equivalent to the atomistic description and in this sense the higher length scales seamlessly merge with the atomic scale.

We note that for a local element the positions of atoms underlying the element are determined entirely by the nodal positions of the element [see (11)]. For nonlocal elements, on the other hand, one needs to know the positions of all atoms underlying those portions of the neighboring elements encompassed by the cut-off circle. Therefore, relations (11) pertaining to all involved elements must be utilized. The computation of $\tilde{u}_{e}$ for the nonlocal element is obviously much more intensive than that for the local element.

To obtain an explicit expression for the stress-tensor elements $T_{\alpha \alpha}$ in terms of the atomic coordinates and interatomic potential $\phi$, we recast the ensemble average in (23). To this end, we invoke the relation (11), which is given in component form by

$$
r_{i \alpha}=\sum_{k=1}^{N_{n}} d_{i k} R_{k \alpha}, \quad \alpha=x, y .
$$

Then using (30), we can rewrite the sum in angular brackets in (23) successively as

$$
\begin{aligned}
\sum_{k=1}^{N_{n}} \frac{\partial U}{\partial R_{k \alpha}} R_{k \alpha} & =\sum_{k=1}^{N_{n}} \sum_{i=1}^{N} \frac{\partial U}{\partial r_{i \alpha}} \frac{\partial r_{i \alpha}}{\partial R_{k \alpha}} R_{k \alpha} \\
& =\sum_{i=1}^{N} \frac{\partial U}{\partial r_{i \alpha}} \sum_{k=1}^{N_{n}} d_{i k} R_{k \alpha} \\
& =\sum_{i=1}^{N} \frac{\partial U}{\partial r_{i \alpha}} r_{i \alpha}, \quad \alpha=x, y .
\end{aligned}
$$

Combining (24), (26), and (27), we can express the potential energy as

$$
U \simeq \sum_{e=1}^{N_{e}} N_{a}^{e} \frac{1}{2} \sum_{j \neq i} \phi\left(r_{i j}\right) .
$$

From (31) and (32) we obtain

$$
\begin{gathered}
\sum_{l=1}^{N} \frac{\partial U}{\partial r_{l \alpha}} r_{l \alpha}=\frac{1}{2} \sum_{e=1}^{N_{e}} N_{a}^{e} \sum_{l=1}^{N} \sum_{j \neq i} \phi^{\prime}\left(r_{i j}\right) \frac{\partial r_{i j}}{\partial r_{l \alpha}} r_{l \alpha}, \\
\alpha=x, y,
\end{gathered}
$$

where $\phi^{\prime}=d \phi / d r$. From (3) we have

$$
\frac{\partial r_{i j}}{\partial r_{l \alpha}}=\frac{\left(r_{i \alpha}-r_{j \alpha}\right)\left(\delta_{i l}-\delta_{j l}\right)}{r_{i j}},
$$

where $\delta$ is the Kronecker symbol. Substituting this expression for $\partial r_{i j} / \partial r_{l \alpha}$ into (33) and carrying out the sum on $l$ yields

$$
\begin{gathered}
\sum_{l=1}^{N} \frac{\partial U}{\partial r_{l \alpha}} r_{l \alpha}=\frac{1}{2} \sum_{e=1}^{N_{e}} N_{a}^{e} \sum_{j \neq i} \phi^{\prime}\left(r_{i j}\right)\left(r_{i \alpha}-r_{j \alpha}\right)^{2} / r_{i j}, \\
\alpha=x, y .
\end{gathered}
$$

Finally, combining (31) and (35), we can rewrite (23) as

$$
\begin{gathered}
T_{\alpha \alpha}=-\frac{N_{n} k_{B} T}{A}+\frac{1}{2 A} \sum_{e=1}^{N_{e}} N_{a}^{e}\left\langle\sum_{j \neq i} \phi^{\prime}\left(r_{i j}\right)\left(r_{i \alpha}-r_{j \alpha}\right)^{2} / r_{i j}\right\rangle, \\
\alpha=x, y,
\end{gathered}
$$

where elements $e$ can be either "local" or "nonlocal." The formula given in (36) is the coarse-grained approximation to the "virial" expression for the stress. When $N=N_{n}$, (36) can be written 


$$
\begin{aligned}
& T_{\alpha \alpha}=-\rho k_{B} T+\frac{1}{2 A} \sum_{i=1}^{2 N} \frac{1}{2} \sum_{j \neq i}\left\langle\phi^{\prime}\left(r_{i j}\right)\left(r_{i \alpha}-r_{j \alpha}\right)^{2} / r_{i j}\right\rangle, \\
& \alpha=x, y,
\end{aligned}
$$

where the index $i$ counts each atom only twice [see discussion below (29)]. The expression in (37) is equivalent to the usual atomic virial expression. ${ }^{28}$

\section{COARSE-GRAINED MONTE CARLO SIMULATIONS}

\section{A. Methods}

We apply the usual Metropolis MC technique ${ }^{29}$ to the coarse-grained system, in which nodal coordinates are the stochastic variables (in lieu of atomic coordinates in the normal procedure) and the configurational energy is given by (24). No unique coarse-graining exists. A virtual infinity of meshes, comprising combinations of elements of various shapes and sizes, could in principle be employed in the coarse-grained MC simulations. However, since the crystal is homogeneous it is reasonable to take all elements to be the same size and shape in the reference configuration $\mathfrak{R}^{0}$. Using strictly congruent equilateral triangles, we can cover the reference configuration exactly with $2^{2 q}$ elements, where $q$ $=2,3,4,5,6$. One-sixth (1/6) of a nodal atom is assigned to each of the six elements to which it is common; one-half $(1 / 2)$ of an atom lying on a side of an element is assigned to each of the two elements which share that side. On account of the p.b.c. only $2^{2 q-1}$ nodes are independent. In Fig. 1, where the special case $q=2$ is depicted, there are 8 independent nodes. Each element includes $N_{a}^{e}=8$ atoms, so the total number of atoms $N=N_{a}^{e} \times 2^{2 q}=128$. In case $2^{2 q-1}=N$, every atom is a node and $N_{e}=2 N$.

For the special mesh to which we restrict our attention in this article, when the crystal is in the reference configuration $\mathfrak{R}^{0}$ (see Fig. 1), the configurational energy is given by

$$
U=\sum_{e=1}^{N_{e}} N_{a}^{e} \widetilde{u}_{e}\left(\Re^{0}\right)=N \widetilde{u}^{0},
$$

where the second equality follows from the equivalence of all elements and all atoms in $\mathfrak{R}^{0}$ and $\widetilde{u}^{0} \equiv \tilde{u}_{e}\left(\mathfrak{R}^{0}\right)$ is the potential energy per atom for the perfect crystal [see (26)]. Now suppose a randomly picked node is subjected to a trial displacement. It is clear that only the configurations of the lattices underlying the elements that share the chosen node change. However, $\tilde{u}_{e}$ changes not only for these six elements, but also for all other nearby elements that are intersected by cutoff circles centered on the centroid atoms of these elements. If the affected elements are labeled by a local index $e^{\prime}$, then the change in the total configurational energy can be written

$$
\Delta U=\sum_{e^{\prime}} N_{a}^{e^{\prime}}\left[\tilde{u}_{e^{\prime}}(\mathfrak{R})-\widetilde{u}^{0}\right]=N_{a}^{e} \sum_{e^{\prime}}\left[\tilde{u}_{e^{\prime}}(\mathfrak{R})-\tilde{u}^{0}\right],
$$

where $\widetilde{u}_{e^{\prime}}$ is given by (26). The second equality of (39) is justified in the current situation where every element contains the same number of atoms $N_{a}^{e}$. The generalization of
(39) to a move from a "current" configuration $\left(\mathfrak{R}^{n-1}\right)$ to a "trial" configuration $\left(\mathfrak{R}^{n}\right)$ can be expressed as

$$
\Delta U=N_{a}^{e} \sum_{e^{\prime}}\left[\widetilde{u}_{e^{\prime}}\left(\Re^{n}\right)-\widetilde{u}_{e^{\prime}}\left(\Re^{n-1}\right)\right] .
$$

For computational efficiency the set of elemental potential energies per atom for the current configuration $\left\{\widetilde{u}_{e^{\prime}}\left(\mathfrak{R}^{n}\right), e\right.$ $\left.=1,2, \ldots, N_{e}\right\}$ is stored. After the $n$th trial move the total potential energy is given in terms of that for the $(n-1)$ th trial move by

$$
U\left(\Re^{n}\right)=U\left(\Re^{n-1}\right)+\Delta U .
$$

If the trial move is rejected, $\Delta U$ is set to 0 . The mean (MC ensemble-average) configurational energy can be written

$$
\langle U\rangle=M^{-1} \sum_{n=1}^{M} U\left(\Re^{n}\right),
$$

where the sum is over $M$ trial moves (configurations).

By the same reasoning the change in the "instantaneous virial" $W_{\alpha}$, defined by

$$
\begin{array}{r}
W_{\alpha}=\frac{1}{2} \sum_{e} N_{a}^{e} \sum_{j \neq i} \frac{\phi^{\prime}\left(r_{i j}\right)\left(r_{i \alpha}-r_{j \alpha}\right)^{2}}{r_{i j}}=\sum_{e} W_{\alpha}^{e}, \\
\alpha=x, y
\end{array}
$$

accompanying the movement of a node is

$$
\Delta W_{\alpha}=\sum_{e^{\prime}}\left[W_{\alpha}^{e^{\prime}}\left(\Re^{n}\right)-W_{\alpha}^{e^{\prime}}\left(\Re^{n-1}\right)\right],
$$

where the contribution to $W_{\alpha}$ of element $e$ is denoted by $W_{\alpha}^{e}$ and is defined implicitly in (43). The sum in (44) is restricted to elements affected by the move, as described above in connection with the computation of the mean potential energy. The virial for the current configuration $\mathfrak{R}^{n}$ is given in terms of that for the immediately preceding configuration by

$$
W_{\alpha}\left(\Re^{n}\right)=W_{\alpha}\left(\Re^{n-1}\right)+\Delta W_{\alpha} .
$$

In this case, as in that of the potential energy, it is computationally more efficient to save the set of current elemental virials $\left\{W_{\alpha}^{e}\left(\mathfrak{R}^{n}\right), e=1,2, \ldots, N_{e} ; \alpha=x, y\right\}$ than to compute them again for each trial move. The MC ensemble-average value of the stress tensor [see (36)] can finally be expressed as

$$
T_{\alpha \alpha}=-N_{n} k_{B} T / A+\left\langle W_{\alpha}\right\rangle / A,
$$

where

$$
\left\langle W_{\alpha}\right\rangle=M^{-1} \sum_{n=1}^{M} W_{\alpha}\left(\Re^{n}\right) .
$$

In the coarse-graining limit $N_{n}=N$, where every atom becomes a node, the coarse-grained Hamiltonian reverts to the original atomic one (i.e., $H_{c}=H$ ). Note, however, that since every element becomes nonlocal in the coarse-graining limit, the burden of computing $\Delta U$ becomes prohibitive [see discussion below (29)]. Consequently, when the coarse- 
TABLE I. Comparison of mean values of configurational energy per atom $\langle U\rangle / N$ and isotropic stress $\tau=\left(T_{x x}+T_{y y}\right) / 2$ for selected thermodynamic states of two-dimensional Lennard-Jonesium. The densities $\rho=0.45,0.55$, and $\rho=0.9165,0.94$ correspond to fluid and solid states, respectively. In present calculation, number of atoms $N=256$; cut-off radius $r_{c}$ is one-half of the shorter side of the system; $M=10^{4} \mathrm{~N}$ configurations used to calculate ensemble averages.

\begin{tabular}{|c|c|c|c|c|c|}
\hline \multicolumn{2}{|c|}{ State } & \multicolumn{2}{|c|}{ Present Work } & \multicolumn{2}{|c|}{ Reference 32} \\
\hline & & $\langle U\rangle$ & & $\langle U\rangle$ & \\
\hline$\rho$ & $T$ & $\bar{N}$ & $\tau$ & $\bar{N}$ & $\tau$ \\
\hline 0.45 & 1.0 & -1.237 & -0.465 & -1.243 & -0.47 \\
\hline 0.55 & 1.0 & -1.503 & -0.705 & -1.500 & -0.70 \\
\hline 0.9165 & 1.0 & -2.530 & -6.488 & -2.529 & -6.49 \\
\hline 0.94 & 1.0 & -2.517 & -7.965 & -2.514 & -7.99 \\
\hline
\end{tabular}

graining limit is reached, we replace the coarse-graining procedure with the atomistic description, ${ }^{29}$ in which atomic positions are the stochastic variables, and by definition we take the values of the properties computed with this atomistic description to be the true (i.e., numerically accurate) values for the equilibrium properties of the crystal. We use these as benchmarks by which to gauge the reliability of the coarsegraining approach.

\section{B. Results}

To test our computer program for the atomistic MC simulations, we attempted to reproduce some existing results for 2D Lennard-Jonesium. Table I shows the comparison for both fluid and solid states. Even though the symmetry of the system dictates $T_{x x}=T_{y y}, T_{x x}$ and $T_{y y}$ were nevertheless computed separately, as an added check on the reliability of the numerical methodology, and found to agree to better than $0.1 \%$ in all cases. Therefore, only the isotropic stress $\tau$ $=\left(T_{x x}+T_{y y}\right) / 2$ is given in Table I. All numerical results there and elsewhere in this article are expressed in the typical dimensionless units based on the parameters of the LennardJones interatomic potential (distance in units of $\sigma$, energy in units of $\epsilon$, stress in units of $\epsilon / \sigma^{2}$ and temperature in units of $\left.\epsilon / k_{B}\right)$. Dimensionless corrections due to cutting off the interatomic potential, given by

$$
\begin{aligned}
U_{\text {corr }} & =\frac{\pi \rho}{r_{c}^{4}}\left[\frac{2}{5 r_{c}^{6}}-1\right], \\
\tau_{\text {corr }} & =-\frac{3 \pi \rho^{2}}{r_{c}^{4}}\left[\frac{4}{5 r_{c}^{6}}-1\right]
\end{aligned}
$$

for the potential energy $\left(U_{\text {corr }}\right)$ and stress $\left(\tau_{\text {corr }}\right)$, respectively, are included in both sets of simulations. We conclude from Table I that the "atomistic" MC program is reliable and can be used to generate benchmarks as required. In the following, we focus on solid states and take the cutoff radius as $r_{c}=2.5$.

For all coarse-grained simulations reported here, we employed a relatively large system $(N=2048)$ and concentrated on the dependence of $\tau$ on the degree of coarse graining [size of the equilateral (reference) triangular element] and the thermodynamic state. All ensemble averages are based on $10^{4}$ MC cycles (i.e., $10^{4} N_{n}$ configurations). In one cycle all nodes are sequentially moved at random just once.

Table II lists the coarse-grained isotropic stress $\tau_{c}$ computed for three densities of the crystal and four temperatures as a function of the "constraining factor"

$$
f_{c}=\left(N-N_{n}\right) / N=1-N_{n} / N,
$$

which we adopt as the measure of coarse graining. The relative percent error in the coarse-grained stress $\tau_{c}$, based on the "atomistic" value $\tau$, is also given in parentheses. As expected, the relative error decreases with decreasing degree of coarse graining (or decreasing size of element) and increases markedly with $T$ at all $\rho$ and $f_{c}$. The absolute error in $\tau_{c}$

$$
\Delta \tau=\tau-\tau_{c},
$$

however, reveals a rather less gloomy picture. In Fig. 2 we plot $\Delta \tau$ as a function of $f_{c}$ (along with a linear regression of each set of data) for all temperatures and densities examined. It is apparent that $\Delta \tau$ decreases linearly with $f_{c}$ for all thermodynamic states. The (negative) rate of variation of $\Delta \tau$ with $f_{c}$ increases rapidly with $T$. In contrast, for significant variations of applied mechanical deformation ( $\pm 10 \%$ changes in $\rho$ ) at fixed $T$, the dependence of $\Delta \tau$ on $f_{c}$ changes little, as indicated by the very similar linear regressions of

TABLE II. Isotropic stress $(\tau)$ for 2D Lennard-Jonesium in various solid states $(\rho, T)$ as a function of degree of coarse graining [measured by $f_{c}$ defined in (49), which takes values $0.996,0.984,0.938,0.750,0.000$, corresponding, respectively, to number of independent nodes $\left.N_{n}=8,32,128,512,2048\right]$. All results are determined by MC simulation. Number in parentheses after entry is percent error based on atomistic value.

\begin{tabular}{llcccccr}
\hline \hline$\rho$ & \multicolumn{1}{c}{$T$} & \multicolumn{1}{c}{8} & \multicolumn{1}{c}{32} & \multicolumn{1}{c}{128} & \multicolumn{1}{c}{512} & \multicolumn{1}{c}{2048} & Atomistic \\
\hline 0.9 & 0.001 & $1.792(0.5)$ & $1.792(0.5)$ & $1.791(0.4)$ & $1.790(0.4)$ & $\ldots$ & 1.783 \\
& 0.01 & $1.792(5.3)$ & $1.791(5.3)$ & $1.785(5.0)$ & $1.769(4.0)$ & $\ldots$ & 1.701 \\
& 0.1 & $1.789(99)$ & $1.778(98)$ & $1.732(92)$ & $1.56(73)$ & $0.90(0.0)$ & 0.90 \\
& 0.2 & $1.786(2451)$ & $1.764(2420)$ & $1.674(2291)$ & $1.34(1814)$ & $\ldots$ & 0.07 \\
1.0 & 0.001 & $-5.570(0.1)$ & $-5.570(0.1)$ & $-5.571(0.1)$ & $-5.572(0.1)$ & $\ldots$ & -5.578 \\
& 0.01 & $-5.570(1.5)$ & $-5.571(1.5)$ & $-5.576(1.4)$ & $-5.591(1.1)$ & $\ldots$ & -5.656 \\
& 0.1 & $-5.573(14)$ & $-5.583(13)$ & $-5.626(13)$ & $-5.79(10)$ & $-6.44(0.0)$ & -6.44 \\
& 0.2 & $-5.576(24)$ & $-5.597(23)$ & $-5.681(22)$ & $-6.01(18)$ & $\ldots$ & -7.29 \\
1.1 & 0.001 & $-22.96(0.04)$ & $-22.96(0.04)$ & $-22.96(0.04)$ & $-22.96(0.04)$ & $\ldots$ & -22.97 \\
& 0.01 & $-22.96(0.4)$ & $-22.96(0.4)$ & $-22.97(0.3)$ & $-22.98(0.3)$ & $\ldots$ & -23.05 \\
& 0.1 & $-22.96(3.7)$ & $-22.97(3.7)$ & $-23.02(3.5)$ & $-23.18(2.8)$ & $-23.85(0.0)$ & -23.85 \\
& 0.2 & $-22.97(7.1)$ & $-22.99(7.0)$ & $-23.07(6.7)$ & $-23.41(5.3)$ & $\cdots$ & -24.73 \\
\hline \hline
\end{tabular}




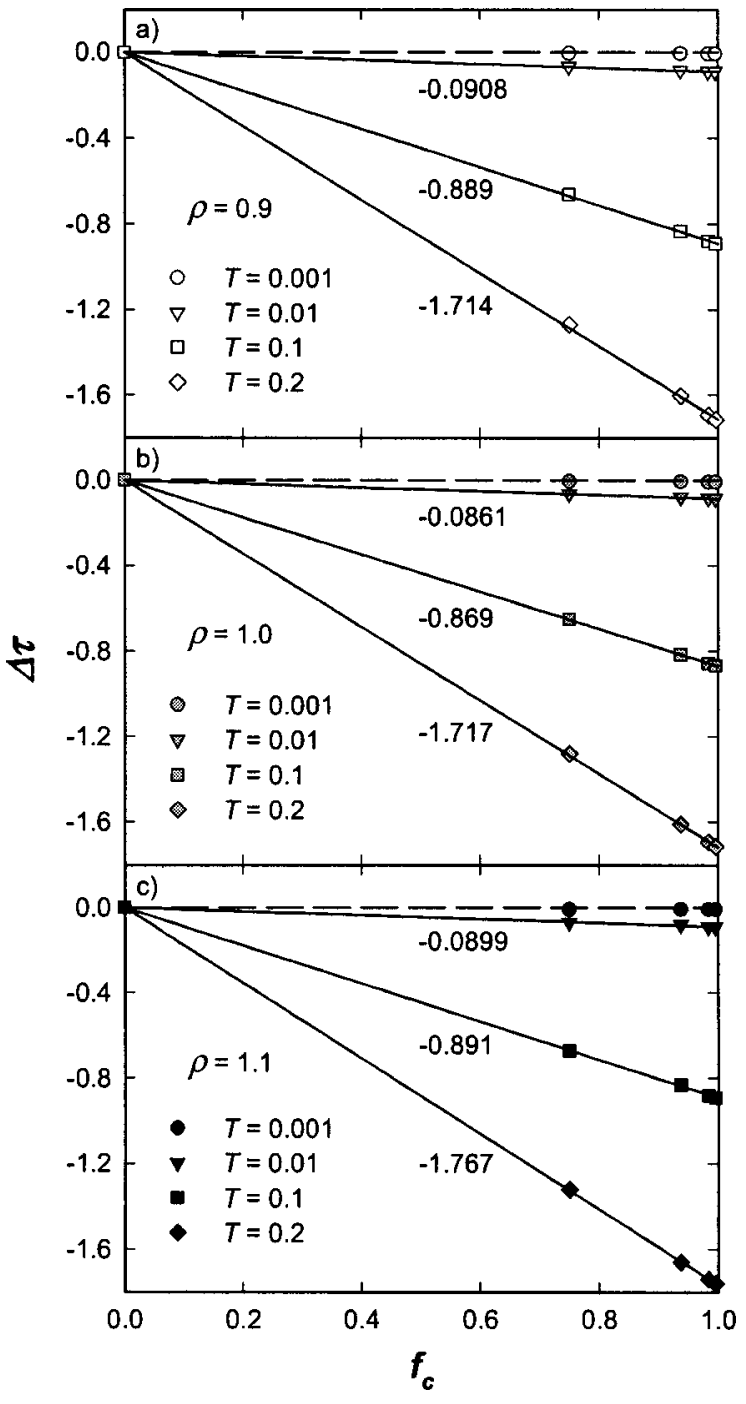

FIG. 2. Absolute error in isotropic stress $\Delta \tau$ [defined in (50)] as a function of constraining factor [defined in (49)] for various thermodynamic states $(\rho, T)$ of 2D Lennard-Jonesium. Solid lines are linear least-squares fits and associated numbers are slopes.

the corresponding data. Furthermore, $\Delta \tau$ is negligibly small (less than $1 \%$ of the reduced unit of stress) for all $f_{c}$ at $T$ $=0.001$ and all densities. Note that the material response at $T=0.001$ is predominantly mechanical. We conclude from these observations that coarse-graining causes noticeable error only in the thermal contribution to the stress.

In terms of $\Delta \tau$ the coarse-graining error at temperatures 0.01 and below is insignificant (less than $10 \%$ of the stress unit) and can be neglected. Note that the triple-point temperature $T_{t}$ of 2D Lennard-Jonesium is about 0.415 and the density of the coexisting solid is about $0.85 .^{30}$ Hence, up to a temperature about an order of magnitude lower than $T_{t}$, the error is tolerable $(\Delta \tau<0.5)$, especially if the stress is high. For a "hard" solid with a melting point in the range 2000 $3000 \mathrm{~K}$ under the ambient pressure, $T_{t}$ is expected to be at least an order of magnitude higher than room temperature. The results presented thus far suggest that the coarsegraining error in the thermal contribution to the stress is not of practical concern for such hard materials at temperatures not much higher than room temperature.

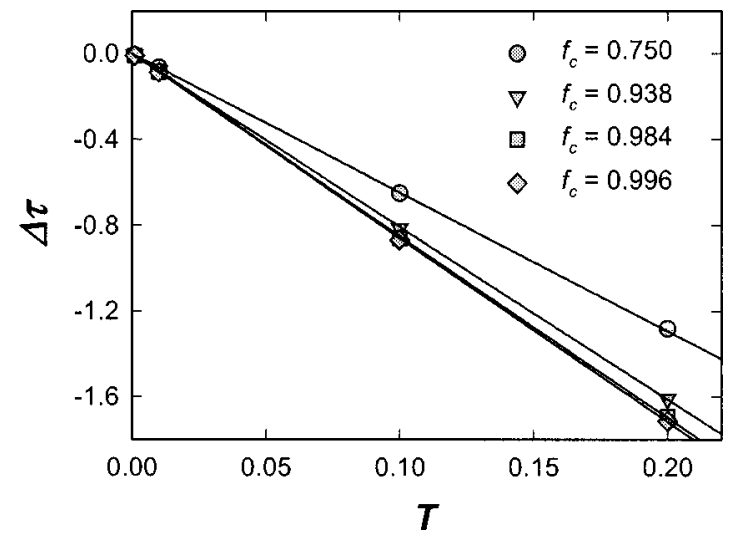

FIG. 3. Absolute error in isotropic stress $(\Delta \tau)$ as function of temperature $(T)$ for 2D Lennard-Jonesium at density $\rho=1.0$ and various degrees of coarse-graining $f_{c}$. Solid lines are linear least-squares fits.

At significantly higher temperatures the error in $\tau$ may become considerable. Figure 3 displays plots of $\Delta \tau$ versus $T$ for all four values of $f_{c}$ and $\rho=1.0$. The following discussion applies, however, to other densities, since $\Delta \tau$ depends only slightly on the mechanical deformation. We find that $\Delta \tau$ depends essentially linearly on $T$ for all $f_{c}$; the slope of the line increases gradually with $f_{c}$. It is clear from (36) that coarsegraining underestimates the ideal-gas contribution to $\tau$ (which is linear in $T$ ). An important question is whether this is the main source of the error seen in Fig. 3. To address this issue, we write $\Delta \tau$ as

$$
\Delta \tau=\Delta \tau_{\mathrm{IG}}+\Delta \tau_{\mathrm{ex}},
$$

where the error in the ideal-gas contribution is

$$
\Delta \tau_{\mathrm{IG}}=-\left(N-N_{n}\right) k_{B} T / A=-\rho f_{c} k_{B} T,
$$

and the error due to the contribution of thermally averaged interactions is

$$
\Delta \tau_{\mathrm{ex}}=\frac{1}{2 A} \sum_{\alpha=x, y}\left[\left\langle\sum_{i=1}^{N} \frac{\partial U}{\partial r_{i \alpha}} r_{i \alpha}\right\rangle-\left\langle\sum_{k=1}^{N_{n}} \frac{\partial U}{\partial R_{k \alpha}} R_{k \alpha}\right\rangle\right] .
$$

From plots of $\Delta \tau_{\mathrm{IG}}$ and $\Delta \tau_{\mathrm{ex}}$ versus $T$ for $\rho=1.0$ and $f_{c}=0.996$ in Fig. 4 it is clear that $\Delta \tau_{\mathrm{ex}}$ swamps $\Delta \tau_{\mathrm{IG}}$. The error in $\Delta \tau$ is, therefore, due primarily to the loss of degrees of freedom over which the gradients of the configurational energy (i.e., the interatomic forces) are thermally averaged.

\section{COARSE GRAINED LOCAL HARMONIC APPROXIMATION}

\section{A. Formula for isotropic stress}

Earlier work by one of us demonstrated that for a pure 1D monatomic crystal treated in the nearest-neighbor harmonic approximation $\Delta \tau$ is exactly linear in $f_{c}$. ${ }^{31}$ The present observation that $\Delta \tau$ for 2D Lennard-Jonesium is essentially linear in $f_{c}$ (see Fig. 2) suggests that the error due to coarse-graining may be estimable in the harmonic approximation. However, the expression for $\Delta \tau$ for the 2D nearestneighbor harmonic model cannot be obtained in enclosed form. We, therefore, introduce the local harmonic approximation (LHA), which has been shown to be viable in other 


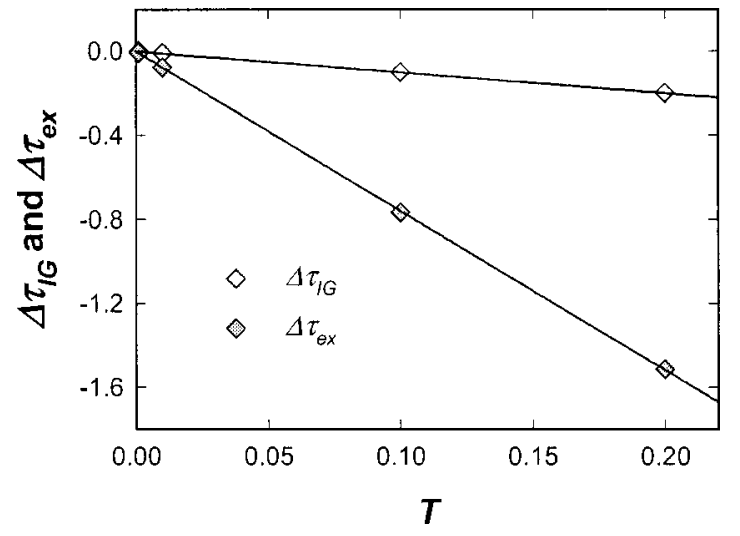

FIG. 4. Ideal-gas $\left(\Delta \tau_{\mathrm{IG}}\right)$ and interaction $\left(\Delta \tau_{\mathrm{ex}}\right)$ contributions to absolute error in isotropic stress as a function of temperature (T) for 2D LennardJonesium at density $\rho=1.0$ and maximum degree of coarse-graining $f_{c}$ $=0.996$. Solid lines are linear least-squares fits.

applications. ${ }^{21-25}$ In essence the LHA neglects couplings between different atoms. The dynamical matrix is therefore block diagonal, consisting of a set of $2 \times 2$ matrices, which can be diagonalized readily to obtain the frequencies of the local atomic vibrations. It is the variation of these frequencies with the density that determines the thermal contribution to the isotropic stress. For simplicity we assume that all elements are local (i.e., all atoms within the cut-off circle lie under the element), although we emphasize that this is not necessary.

Expanding the coarse-grained potential energy in Taylor's series and neglecting terms of degree higher than the second in the LHA, we obtain

$$
\begin{aligned}
U\left(\mathbf{R}_{1}, \mathbf{R}_{2}, \ldots, \mathbf{R}_{N_{n}}\right) & \\
\approx & U^{0}+\sum_{k=1}^{N_{n}}\left[\frac{1}{2}\left(\frac{\partial^{2} U}{\partial X_{k}^{2}}\right)_{0} \Delta X_{k}^{2}+\left(\frac{\partial^{2} U}{\partial X_{k} \partial Y_{k}}\right)_{0} \Delta X_{k} \Delta Y_{k}\right. \\
& \left.+\frac{1}{2}\left(\frac{\partial^{2} U}{\partial Y_{k}^{2}}\right)_{0}^{\Delta Y_{k}^{2}}\right],
\end{aligned}
$$

where $U^{0}=U\left(\mathbf{R}_{1}^{0}, \mathbf{R}_{2}^{0}, \ldots, \mathbf{R}_{N_{n}}^{0}\right)$ stands for the potential energy of the crystal in its equilibrium configuration (denoted by super- and subscript zeroes) and $\Delta \alpha_{i}=\alpha_{i}-\alpha_{i}^{0}(\alpha=X, Y)$ stands for Cartesian components of the displacement of node $i$ from its equilibrium position. From (24), (27), and (30), we obtain the following expressions:

$$
\begin{aligned}
\left(\frac{\partial^{2} U}{\partial X_{k}^{2}}\right)_{0}=\sum_{e}{ }^{\prime} \frac{1}{2} N_{a}^{e} \sum_{j \neq i}\left[\frac{\phi^{\prime \prime}\left(r_{i j}^{0}\right)}{\left(r_{i j}^{0}\right)^{2}}\left(x_{i j}^{0}\right)^{2}\left(d_{i k}-d_{j k}\right)^{2}\right. \\
\left.+\frac{\phi^{\prime}\left(r_{i j}^{0}\right)}{\left(r_{i j}^{0}\right)^{3}}\left(y_{i j}^{0}\right)^{2}\left(d_{i k}-d_{j k}\right)^{2}\right], \\
\left(\frac{\partial^{2} U}{\partial X_{k} \partial Y_{k}}\right)_{0}=\sum_{e}{ }^{\prime} \frac{1}{2} N_{a}^{e} \sum_{j \neq i}\left[\frac{\phi^{\prime \prime}\left(r_{i j}^{0}\right)}{\left(r_{i j}^{0}\right)^{2}} x_{i j}^{0} y_{i j}^{0}\left(d_{i k}-d_{j k}\right)^{2}\right. \\
\left.-\frac{\phi^{\prime}\left(r_{i j}^{0}\right)}{\left(r_{i j}^{0}\right)^{3}} x_{i j}^{0} y_{i j}^{0}\left(d_{i k}-d_{j k}\right)^{2}\right],
\end{aligned}
$$

$$
\begin{aligned}
\left(\frac{\partial^{2} U}{\partial Y_{k}^{2}}\right)_{0}= & \sum_{e}{ }^{\prime} \frac{1}{2} N_{a}^{e} \sum_{j \neq i}\left[\frac{\phi^{\prime \prime}\left(r_{i j}^{0}\right)}{\left(r_{i j}^{0}\right)^{2}}\left(y_{i j}^{0}\right)^{2}\left(d_{i k}-d_{j k}\right)^{2}\right. \\
& \left.+\frac{\phi^{\prime}\left(r_{i j}^{0}\right)}{\left(r_{i j}^{0}\right)^{3}}\left(x_{i j}^{0}\right)^{2}\left(d_{i k}-d_{j k}\right)^{2}\right] .
\end{aligned}
$$

The prime on the summation on $e$ indicates it is restricted to elements having node $k$ in common.

The evaluation of the expressions in (55) is aided by reference to Fig. 1. We focus first on element I and compute the coefficients $d_{i k}$ for the indicated centroid atom $i$. Inscribing the appropriate triangles [see relation (10)], we find

$$
d_{i k}-d_{j k}=0,
$$

for neighbors $j$ in the same horizontal row as $i$ (e.g., nearestneighbor atoms 1 and 4) and

$$
d_{i k}-d_{j \pm q, k}= \pm q /(p+1),
$$

for neighbors $j \pm q$ that are either $q$ rows above $i(+)$ (e.g., atoms 5 and 6) or $q$ rows below $i(-)$ (e.g., atoms 2 and 3 ). In (57) $p$ is the number of atoms along a side of I, exclusive of the atoms at the vertices. Utilizing the rules in (56) and (57), which can be generalized to the other elements, we deduce the following relations:

$$
\begin{aligned}
& \sum_{e}^{\prime} \sum_{j=1}^{6}\left(x_{i j}^{0}\right)^{2}\left(d_{i k}-d_{j k}\right)^{2}=\frac{12 a^{2}}{(p+1)^{2}}, \\
& \sum_{e}^{\prime} \sum_{j=1}^{6} x_{i j}^{0} y_{i j}^{0}\left(d_{i k}-d_{j k}\right)^{2}=0, \\
& \sum_{e}^{\prime} \sum_{j=1}^{6}\left(y_{i j}^{0}\right)^{2}\left(d_{i k}-d_{j k}\right)^{2}=\frac{12 a^{2}}{(p+1)^{2}},
\end{aligned}
$$

where the sums on $j$ are restricted to the six nearest neighbors of the centroid atom. Combining (55) and (58), we obtain

$$
\kappa_{c}=\left(\frac{\partial^{2} U}{\partial X_{k}^{2}}\right)_{0}=\left(\frac{\partial^{2} U}{\partial Y_{k}^{2}}\right)_{0}=3\left[\phi^{\prime \prime}(a)+\phi^{\prime}(a) / a\right] .
$$

In arriving at (59) we invoke the relation

$$
N_{a}^{e}=(p+1)^{2} / 2 \text {. }
$$

The total potential energy can now be written as

$$
U\left(\mathbf{R}_{1}, \mathbf{R}_{2}, \ldots, \mathbf{R}_{N_{n}}\right)=U^{0}+\frac{1}{2} \sum_{k=1}^{N_{n}} m_{c} \omega_{c}^{2}\left(\mathbf{R}_{k}-\mathbf{R}_{k}^{0}\right)^{2},
$$

where the coarse-grained fundamental frequency $\omega_{c}$ is given by

$$
\omega_{c}^{2}=\kappa_{c} / m_{c},
$$

and $m_{c}$ is the effective mass associated with the node. Within the framework of the LHA, the coarse-grained crystal is equivalent to a set of $N_{n}$ isotropic harmonic oscillators, for which the classical-limit Helmholtz potential ${ }^{21}$ is

$$
F_{c, \mathrm{LHA}}=U^{0}-2 N_{n} k_{B} T \ln \left(k_{B} T / h\right)+N_{n} k_{B} T \ln \omega_{c}^{2},
$$

and the coarse-grained isotropic stress is 
TABLE III. Isotropic stress $\left(\tau_{c, \text { LHA }}\right)$ for $2 \mathrm{D}$ Lennard-Jonesium in the LHA. Conditions are same as those detailed in caption of Table II.

\begin{tabular}{|c|c|c|c|c|c|c|c|}
\hline$\rho$ & $T$ & 8 & 32 & 128 & 512 & 2048 & $\tau_{\text {LHA }}$ \\
\hline \multirow[t]{4}{*}{0.9} & 0.001 & 1.598 & 1.597 & 1.597 & 1.595 & 1.588 & 1.588 \\
\hline & 0.01 & 1.597 & 1.596 & 1.592 & 1.574 & 1.504 & 1.504 \\
\hline & 0.1 & 1.594 & 1.583 & 1.539 & 1.365 & 0.666 & 0.666 \\
\hline & 0.2 & 1.590 & 1.568 & 1.481 & 1.132 & -0.266 & -0.266 \\
\hline \multirow[t]{4}{*}{1.0} & 0.001 & -5.810 & -5.810 & -5.811 & -5.812 & -5.819 & -5.819 \\
\hline & 0.01 & -5.810 & -5.811 & -5.816 & -5.832 & -5.899 & -5.899 \\
\hline & 0.1 & -5.813 & -5.824 & -5.865 & -6.032 & -6.698 & -6.698 \\
\hline & 0.2 & -5.817 & -5.838 & -5.921 & -6.254 & -7.585 & -7.585 \\
\hline \multirow[t]{4}{*}{1.1} & 0.001 & -23.25 & -23.25 & -23.25 & -23.25 & -23.26 & -23.26 \\
\hline & 0.01 & -23.25 & -23.25 & -23.26 & -23.27 & -23.34 & -23.34 \\
\hline & 0.1 & -23.25 & -23.26 & -23.31 & -23.48 & -24.15 & -24.15 \\
\hline & 0.2 & -23.26 & -23.28 & -23.36 & -23.70 & -25.06 & -25.06 \\
\hline
\end{tabular}

$$
\begin{aligned}
\tau_{c, \mathrm{LHA}} & =\left(\partial F_{c, \mathrm{LHA}} / \partial A\right)_{T, N_{n}} \\
& =\frac{1}{N \sqrt{3} a} \frac{\partial F_{c, \mathrm{LHA}}}{\partial a}=\frac{1}{N \sqrt{3} a}\left[\frac{\partial U^{0}}{\partial a}+\frac{N_{n} k_{B} T}{\omega_{c}^{2}} \frac{\partial \omega_{c}^{2}}{\partial a}\right],
\end{aligned}
$$

where the second line depends on (5). From (24), (26), and (27), we obtain

$$
\begin{aligned}
U^{0} & =\frac{1}{2} \sum_{e=1}^{N_{e}} N_{a}^{e} \sum_{i \neq j} \phi\left(r_{i j}^{0}\right) \\
& \approx 3 N[\phi(a)+\phi(\sqrt{3} a)+\phi(2 a)],
\end{aligned}
$$

where we include three nearest-neighbor sets in the sum on $j$. Evaluating the required partial derivatives of $\omega_{c}^{2}$ and $U^{0}$ from (59), (62), and (65) and plugging the results into (64) gives

$$
\begin{aligned}
\tau_{c, \mathrm{LHA}}= & \frac{\sqrt{3}}{a}\left[\phi^{\prime}(a)+\sqrt{3} \phi^{\prime}(\sqrt{3} a)+2 \phi^{\prime}(2 a)\right] \\
& +\frac{\sqrt{3} k_{B} T N_{n}}{3 a N} \frac{\phi^{\prime \prime \prime}(a)+\phi^{\prime \prime}(a) / a-\phi^{\prime}(a) / a^{2}}{\phi^{\prime \prime}(a)+\phi^{\prime}(a) / a} .
\end{aligned}
$$

Although we derived the formula in (66) under the restriction that the elements are local, we nevertheless assume it to be valid for nonlocal elements for purposes of comparison with the "exact" MC results in Sec. VB. Note that in the atomistic limit $\left(N_{n} \rightarrow N\right)$, (66) becomes

$$
\begin{aligned}
\tau_{\mathrm{LHA}}= & \frac{\sqrt{3}}{a}\left[\phi^{\prime}(a)+\sqrt{3} \phi^{\prime}(\sqrt{3} a)+2 \phi^{\prime}(2 a)\right] \\
& +\frac{\sqrt{3} k_{B} T}{3 a} \frac{\phi^{\prime \prime \prime}(a)+\phi^{\prime \prime}(a) / a-\phi^{\prime}(a) / a^{2}}{\phi^{\prime \prime}(a)+\phi^{\prime}(a) / a} .
\end{aligned}
$$

This expression can be derived by applying the LHA directly to the original fully atomistic Hamiltonian [(4)].

\section{B. Results}

Table III lists $\tau_{\text {LHA }}[(67)]$ for the same thermodynamic states as Table II. The discrepancies between LHA and "atomistic" MC are only about $1 \%$ at $\rho=1.1$ and $4 \%$ at $\rho$ $=1.0$, irrespective of temperature. For these states the LHA is in reasonably good agreement with MC. However, at the lowest density $(\rho=0.9)$, at which the crystal is under tension, the LHA does not fare so well. The error increases with $T$ and at the highest temperature $(T=0.2)$ the LHA predicts the crystal to be under compression.

Table III also lists $\tau_{c, \text { LHA }}[(66)]$ as a function of $f_{c}$. In parallel with Table II, the relative error in $\tau_{c \text {. LHA }}$, based on $\tau_{\mathrm{LHA}}$, decreases with decreasing $f_{c}$, or decreasing size of element, and increases strongly with $T$ for all $\rho$ and $f_{c}$. Thus, Table III essentially corroborates Table II.

From (66) and (67) we compute the absolute error due to coarse-graining within the LHA

$$
\begin{aligned}
\Delta \tau_{\mathrm{LHA}} & =\tau_{\mathrm{LHA}}-\tau_{c, \mathrm{LHA}} \\
& =\frac{\sqrt{3} f_{c} k_{B} T}{3 a} \frac{\phi^{\prime \prime \prime}(a)+\phi^{\prime \prime}(a) / a-\phi^{\prime}(a) / a^{2}}{\phi^{\prime \prime}(a)+\phi^{\prime}(a) / a} .
\end{aligned}
$$

In Fig. 5 we plot $\Delta \tau_{\text {LHA }}$ versus $f_{c}$ for the data in Tables II and III, all of which refer to $T=0.1$ and the several densities examined. We also plot in Fig. $6 \Delta \tau_{c}$ versus $T$ for fixed $N_{n}$ $=128\left(f_{c}=0.9375\right)$. The plots exhibited in Figs. 5 and 6

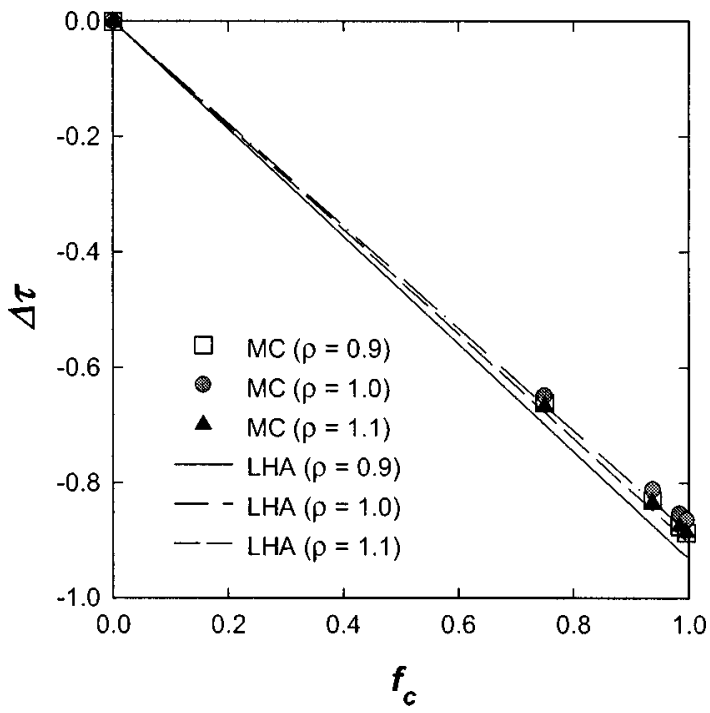

FIG. 5. Error in isotropic stress, $\Delta \tau$, as function of degree of coarsegraining, $f_{c}$, at fixed temperature $T=0.1$ and various densities. Discrete points correspond to MC results and lines to LHA Eq. (68). 


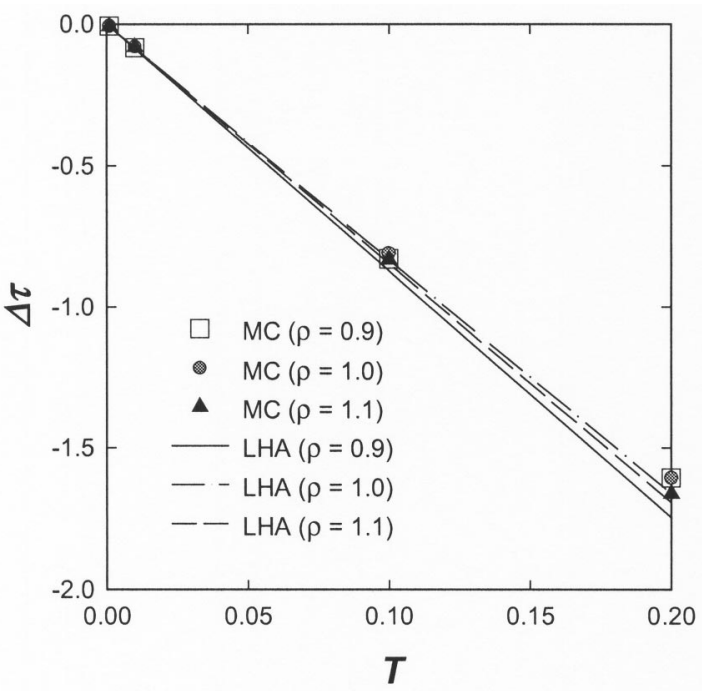

FIG. 6. Error in the isotropic stress, $\Delta \tau$, as function of temperature $(T)$ at fixed degree of coarse-graining $f_{c}=0.9375\left(N_{n}=128\right)$ and various densities. Labels are same as those of Fig. 5.

verify that the LHA not only provides a rationale for the linearity of $\Delta \tau_{c}$ in $T$ and $f_{c}$, but also provides a good quantitative estimate of $\Delta \tau_{c}$.

\section{CONCLUSION}

In this paper, the $\mathrm{QC}$ technique is extended to nonzero temperatures. A coarse-grained Hamiltonian is derived for the nodes of the mesh, which behave as quasiparticles whose interactions are mediated by the underlying atoms constrained to move in unison with the nodes. The coarsegrained isotropic stress is computed by means of the Monte Carlo method. The procedure was applied to a simple model: A pure single crystal of two-dimensional Lennard-Jonesium. In comparison with the exact (noncoarse-grained) isotropic stress for various thermodynamic states, coarse-graining leads to an error for isotropic stress. The error in the coarsegrained isotropic stress depends linearly on the degree of coarse-graining and on the absolute temperature. The linear dependencies are nicely rationalized by applying the LHA to the coarse-grained Hamiltonian. The error is due to the complete neglect of the thermal motions of non-nodal atoms underlying the finite-element mesh. The thermal vibrations of these atoms are frozen out by the constraint that they move in lockstep with the nodes.

We emphasize that all of the above results pertain to the highly idealized two-dimensional system involving only two-body interactions and subject to homogeneous isotropic deformations. Whether the dependencies of coarse-graining errors on $f_{c}, \rho$, and $T$ are as simple for more realistic models of materials under more general loading conditions remains to be determined.

\section{ACKNOWLEDGMENTS}

We thank the National Science Foundation for generous support through the Grant Programs ECS-9900127, ITR0112929, and NIRT-0210850. We also thank UNL research computing facility for assisting us in the computation.

${ }^{1}$ H. Gleiter, Prog. Mater. Sci. 33, 223 (1989).

${ }^{2}$ M. Weins, B. Chalmers, H. Gleiter, and M. Ashby, Scr. Metall. 3, 601 (1969).

${ }^{3}$ A. I. Mikhailin and A. E. Romanov, Sov. Phys. Solid State 28, 337 (1986).

${ }^{4}$ J. Schiotz, L. M. Canel, and A. E. Carlsson, Phys. Rev. B 55, 6211 (1997).

${ }^{5}$ J. Schiotz and A. E. Carlsson, Philos. Mag. A 80, 69 (2000).

${ }^{6}$ A. Nakano, R. K. Kalia, and P. Vashishta, Comput. Phys. Commun. 83, 197 (1994).

${ }^{7}$ P. Vashishta, A. Nakano, R. K. Kalia, and I. Ebbsjö, Mater. Sci. Eng., B 37, 56 (1996).

${ }^{8}$ M. E. Bachlechner, A. Omeltchenko, A. Nakano, R. K. Kalia, P. Vashishta, I. Ebbsjö, and A. Madhukar, Phys. Rev. Lett. 84, 322 (2000).

${ }^{9}$ F. F. Abraham, D. Schneider, B. Land, D. Lifka, J. Skovira, J. Gerner, and M. Rosendrantz, J. Mech. Phys. Solids 45, 1461 (1997).

${ }^{10}$ V. Bulatov, F. F. Abraham, L. P. Kubin, L. Devincre, and S. Yip, Nature (London) 391, 669 (1998).

${ }^{11}$ S. Kohlhoff, P. Gumbsch, and H. F. Fischmeister, Philos. Mag. A 64, 851 (1991).

${ }^{12}$ F. F. Abraham, J. Q. Broughton, N. Bernstein, and E. Kaxiras, Comput. Phys. 12, 538 (1998).

${ }^{13}$ F. F. Abraham, J. Q. Broughton, N. Bernstein, and E. Kaxiras, Europhys. Lett. 44, 783 (1998).

${ }^{14}$ J. Q. Broughton, F. F. Abraham, N. Bernstein, and E. Kaxiras, Phys. Rev. B 60, 2391 (1999).

${ }^{15}$ R. E. Rudd and J. Q. Broughton, Phys. Rev. B 58, R5893 (1998); R. E. Rudd and J. Q. Broughton, Phys. Status Solidi B 217, 251 (2000).

${ }^{16}$ E. B. Tadmor, M. Ortiz, and R. Phillips, Philos. Mag. A 73, 1529 (1996).

${ }^{17}$ E. B. Tadmor, G. S. Smith, N. Bernstein, and E. Kaxiras, Phys. Rev. B 59, 235 (1999).

${ }^{18}$ E. B. Tadmor, R. Philips, and M. Ortiz, Int. J. Solids Struct. 37, 379 (2000).

${ }^{19}$ V. B. Shenoy, R. Miller, E. B. Tadmor, R. Philips, and M. Ortiz, Phys. Rev. Lett. 80, 742 (1998); V. B. Shenoy, R. Miller, E. B. Tadmor, D. Rodney, R. Phillips, and M. Ortiz, J. Mech. Phys. Solids 47, 611 (1999).

${ }^{20}$ R. Phillips, M. Dittrich, and K. Schulten, Ann. Rev. Mater. Res. 32, 219 (2002).

${ }^{21}$ R. LeSar, R. Najafabadi, and D. J. Srolovitz, Phys. Rev. Lett. 63, 624 (1989).

${ }^{22}$ A. P. Sutton, Philos. Mag. A 60, 147 (1989).

${ }^{23}$ A. P. Sutton, Philos. Trans. R. Soc. London, Ser. A 341, 233 (1992).

${ }^{24}$ S. M. Foiles, Phys. Rev. B 49, 14930 (1994).

${ }^{25}$ J. Rickman and R. LeSar, Ann. Rev. Mater. Res. 32, 195 (2002).

${ }^{26}$ J. H. Weiner, Statistical Mechanics of Elasticity (Wiley, New York, 1983), Chap. 1.

${ }^{27}$ O. C. Zienkiewicz and R. L. Taylor, The Finite Element Method, 5th ed. (Butterworth-Heinemann, Oxford, 2000), Vol. I, Chap. 4.

${ }^{28}$ D. A. McQuarrie, Statistical Mechanics (Harper Collins, New York, 1976), Chap. 13

${ }^{29}$ M. P. Allen and D. J. Tildesley, Computer Simulation of Liquids (Clarendon, Oxford, 1989), Chap. 4.

${ }^{30}$ F. F. Abraham, Phys. Rep. 80, 339 (1981).

${ }^{31}$ D. J. Diestler, Phys. Rev. B 66, 184104 (2002).

${ }^{32}$ J. A. Barker, D. Henderson, and F. F. Abraham, Physica A 106, 226 (1981). 